\title{
CARACTERÍSTICAS EMPRENDEDORAS EN ESTUDIANTES DE ENFERMERÍA
}

ENTERPRISING CHARACTERISTICS IN NURSING STUDENTS

\author{
PÁRAMO GUTIÉRREZ GUADALUPE LIZBETH', PÉREZ MONJARÁS JUAN DIEGO', PÉREZ \\ URBINA LUCERO ${ }^{1}$, TREJO PITAYO EDUARDO ${ }^{1}$, ÁLVAREZ AGUIRRE ALICIA ${ }^{2^{*}}$
}

\section{ABSTRACT}

Introduction: Enterprising is the individual behavior that tends to the challenge, to create new conditions and improve existing realities, aiming at the pursuit of an independent career in the future. Objective: To know the enterprising characteristics of nursing students. Methodology: Descrip tive transversal study, involving 188 nursing students from a Higher Education Institution in Guanajuato, Mexico, available at the time of the data collection, who responded to the Enterprising Attitude Scale, for the data analysis descriptive statistics was obtained, we had the informed consent from the participants. Results: $77.1 \%$ were women, with an age range between 18 to 26 years old, $50.5 \%$ reported having work experience. In relation to enterprising characteristics, $36.2 \%$ refer to aim for excellence in everything they do, $64.5 \%$ consider that everything is possible if they believe they can do it, $68.6 \%$ always try to learn from their own mistakes, $37.8 \%$ are able to imagine how things can be done to work. Conclusion: The results show the enterprising characteristics of the participants in which stand out to have confidence in their projects, excellence in everything they do, among others. For this reason, this study gives indications of the emphasis on the for mation that nursing professionals oriented to enterprising should have, to favor an autonomous practice.

Key words: Professional education, nursing students, nursing (DeCS, BIREME).

\section{RESUMEN}

ntroducción: El emprendimiento es la conducta individual que tiende al reto, para crear nuevas condiciones y mejorar las realidades existentes, apuntando al seguimiento de una carrera independiente en el futuro. Objetivo: Conocer las características emprendedoras de los estudiantes de enfermería. Metodología: Estudio transversal descriptivo, participaron 188 estudiantes de enfermería, de una Institución de Educación Superior en Guanajuato, México, disponibles en el momento de la colecta de información, quienes dieron respuesta a la Escala de Actitud Emprendedora, para el análisis de los datos se obtuvo estadística descriptiva, se contó con el consentimiento informado de los participantes. Resultados: $77.1 \%$ fueron mujeres, con rango de edad entre 18 y 26 años, 50.5\% refirió tener experiencia laboral. En relación a las características de emprendimiento $36.2 \%$ refiere apuntar a la excelencia en cada cosa que hace, $64.5 \%$ considera que todo es posible sí cree poder hacerlo, $68.6 \%$ siempre intenta aprender lecciones de sus propios errores, $37.8 \%$ son capaces de imaginar cómo pueden hacerse funcionar las cosas. Conclusión: Los resultados dan cuenta de las características emprendedoras de los participantes entre las que se destacan tener confianza en sus proyectos, la excelencia en cada cosa que hacen, entre otras. Por tal motivo, este estudio da indicios del énfasis que debe tener la formación de los profesionales de enfermería orientado al emprendimiento, a fin de favorecer una práctica autónoma.

Palabras claves: Educación profesional, estudiantes de enfermería, enfermería (DeCS).

Estudiante de Licenciatura en Enfermería y Obstetricia, División de Ciencias de la Salud e Ingenierías, Campus Celaya-Salvatierra, Universidad de Guanajuato.

${ }^{2 *}$ Doctora en Ciencias de Enfermería. Departamento de Enfermería Clínica. División de Ciencias de la Salud e Ingenierías, Campus Celaya-Salvatierra. Universidad de Guanajuato, E-mail: alicia. alvarez@ugto.mx 


\section{INTRODUCCIÓN}

El emprendimiento es una cualidad y habilidad del individuo que aprueba iniciar retos, proyectos; permite avanzar e ir más allá de lo alcanzado(1), desarrolla insatisfacción con quien es y sus logros, generando mayores $\operatorname{logros}^{(2)}$. Los países más competitivos y las economías más desarrolladas se distinguen por contar con un tejido productivo que tiene su origen en un emprendimiento inteligente, ya que está basado en el uso innovador del conocimiento y la creatividad, y en tecnologías avanzadas, que muestra además gran capacidad de mercado y de internacionalización ${ }^{(3)}$.

El estudio del emprendimiento es de relevancia, es una herramienta para el desarrollo económico y generación de empleo en todos los países, lo que da origen a una mayor cantidad de oportunidad laboral para la fuerza de trabajo y con ello un mejor nivel de bienestar económico en la población ${ }^{(4-5)}$. Las universidades juegan un papel primordial en el logro de esos objetivos. Por tanto, la formación emprendedora debe dotar a los estudiantes universitarios del conocimiento para desarrollar comportamientos, habilidades y capacidades para crear valor en la sociedad ${ }^{(1)}$.

Además uno de los aspectos fundamentales de la universidad en la sociedad del conocimiento es que promueve y adopta nuevos roles y actividades lejos de sus tradicionales funciones de formación, tales como fomento del espíritu emprendedor, transferencia del conocimiento, formación continua e innovación, así como la vinculación universidad - empresa que posicionan a la institución educativa como líder en las transformaciones de éxito para mayor impacto económico, social y cultural de la sociedad(6).

El emprendimiento es una actividad humana compleja, en ella convergen factores socio personales, cognitivos, económicos, políticos y culturales tales como el sistema de valores del individuo, cultura, su entorno social, familiar y educativo, así como la autoeficacia, estos factores pueden conformar el deseo de, crear o no, una iniciativa de emprendimiento(5-7). Además la evidencia empírica asocia las características de autocontrol, asumir riesgos, autoconfianza, necesidad de logro, tolerancia a la ambigüedad y capacidad de innovación ${ }^{(8)}$ con el emprendimiento. En este sentido la educación debe consistir en la generación de competencias de emprendimiento, que desarrolle rasgos que se asocien con el éxito de su Licenciatura e interés a emprender en el ámbito laboral ${ }^{(9)}$.

Los programas de formación de Licenciados en Enfermería favorecen prácticas emprendedoras seguidas de crecientes desafíos, en medio de los múltiples espacios y posibilidades de actuación de los profesionales de enfermería entre los que destacan: 1) En la promoción de la salud: los consultorios y servicios que promuevan una mejora del bienestar de la población; 2) En la recuperación de la salud: los servicios en hospitales y domicilios, además de las prácticas dirigidas al cuidado especial de niños y ancianos ${ }^{(10)}$. Por lo que las instituciones formadoras además de preparar a los futuros profesionales de enfermería para el trabajo, los incentivan a buscar nuevas alternativas, como escoger nuevos espacios de trabajo, descubrir nuevas formas de ocupar su espacio, comprender el arte de crear sus propias acciones emprendedoras, entre otros ${ }^{(10)}$.

Las características emprendedoras en los estudiantes de enfermería favorecen en ellos las herramientas necesarias para promover una actitud de vivir saludable en el individuo, familia y comunidad a través de procesos interactivos con miras a su emancipación como protagonistas de su trayectoria escolar y profesional ${ }^{(11)}$.

La evidencia empírica reportó que $57 \%$ de los participantes de un estudio realizado en estudiantes universitarios afirmaron poseer intención emprendedora ${ }^{(7)}$. Otra investigación refirió que los estudiantes que tienen voluntad de emprender son quienes consideran tener los conocimientos, habilidades y experiencia para poner en marcha un nuevo negocio y pertenecían a familias de menores ingresos ${ }^{(5)}$. Respecto a la diferencia por género un estudio reportó que no existe diferencia en las actitudes emprendedoras entre estudiantes hombres y mujeres ${ }^{(12)}$. En una investigación realizada en el área de enfermería identificó que las licenciadas en ejercicio libre se caracterizan por su libertad, su autonomía y por proveer un cuidado integral y humanizado ${ }^{(13)}$. El impacto de este estudio radica en evidenciar las características emprendedoras de los estudiantes de enfermería a fin de contribuir en una práctica autónoma. El objetivo del estudio fue conocer las características emprendedoras de los estudiantes de enfermería.

\section{METODOLOGÍA}

Estudio transversal descriptivo, participaron 188 estudiantes, por muestreo intencional, que cursaban la Licenciatura en Enfermería y Obstetricia en una Institución de Educación Superior en Guanajuato, México, en el periodo Agosto-Diciembre de 2017.

Las características emprendedoras se midieron con la Escala Multidimensional ${ }^{(14)}$, conformada por 15 reactivos con respuestas tipo Likert (totalmente en desacuerdo 1, a totalmente en desacuerdo 4), la evaluación de la escala es a mayor puntaje mayor características emprendedoras. La escala reporta 0.86 de confiabilidad mediante Alfa de Cronbach.

Previo a la colecta de datos se contó con la autorización de los participantes, a través del consentimiento informado, a quienes se les comentó sobre los objetivos del proyecto y la importancia de su participación.

El análisis de datos se obtuvo con estadística descriptiva con apoyo del programa estadístico SPSS versión 17 , se usó fre- 
Tabla 1. Características emprendedoras de los participantes del estudio

\begin{tabular}{|c|c|c|c|c|c|c|c|c|}
\hline \multirow[t]{2}{*}{ Reactivo } & \multicolumn{2}{|c|}{$\begin{array}{l}\text { Totalmente } \\
\text { en desacuer- } \\
\text { do }\end{array}$} & \multicolumn{2}{|c|}{$\begin{array}{l}\text { Desacuerdo } \\
\text { en algo }\end{array}$} & \multicolumn{2}{|c|}{$\begin{array}{l}\text { De acuerdo } \\
\text { en algo }\end{array}$} & \multicolumn{2}{|c|}{$\begin{array}{l}\text { Totalmente } \\
\text { de acuerdo }\end{array}$} \\
\hline & $f$ & $\%$ & $f$ & $\%$ & $f$ & $\%$ & $f$ & $\%$ \\
\hline $\begin{array}{l}\text { P1.Cuando tengo un proyecto, tengo } \\
\text { confianza en poder alcanzar el éxito. }\end{array}$ & 1 & 0.5 & 16 & 8.5 & 93 & 49.5 & 78 & 41.5 \\
\hline $\begin{array}{l}\text { P2.Apunto a la excelencia en cada cosa } \\
\text { que hago. }\end{array}$ & 3 & 1.6 & 24 & 12.8 & 93 & 49.5 & 68 & 36.2 \\
\hline $\begin{array}{l}\text { P3. Me gusta darme retos cuando } \\
\text { emprendo un nuevo proyecto. }\end{array}$ & 3 & 1.6 & 8 & 4.3 & 86 & 45.7 & 91 & 48.4 \\
\hline $\begin{array}{l}\text { P4. Intento ser mejor en mi área de } \\
\text { competencia. }\end{array}$ & 3 & 1.6 & 12 & 6.4 & 69 & 36.7 & 104 & 55.3 \\
\hline $\begin{array}{l}\text { P5. Estoy siempre dispuesto a } \\
\text { emprender nuevos proyectos. }\end{array}$ & 5 & 2.7 & 18 & 9.6 & 85 & 45.2 & 80 & 42.6 \\
\hline $\begin{array}{l}\text { P6. Cuando enfrento dificultades, busco } \\
\text { soluciones alternativas. }\end{array}$ & 6 & 3.2 & 6 & 3.2 & 68 & 36.2 & 108 & 57.4 \\
\hline $\begin{array}{l}\text { P7. Siempre intento aprender lecciones } \\
\text { de mis propios errores. }\end{array}$ & 4 & 2.1 & 5 & 2.7 & 50 & 26.6 & 129 & 68.6 \\
\hline $\begin{array}{l}\text { P8. Siempre doy lo mejor de mí en todo } \\
\text { lo que hago }\end{array}$ & 4 & 2.1 & 8 & 4.3 & 64 & 34.0 & 112 & 59.6 \\
\hline P9. Todo es posible si creo en hacerlo. & 4 & 2.1 & 5 & 2.7 & 56 & 29.8 & 123 & 65.4 \\
\hline $\begin{array}{l}\text { P10. Después de un error, soy capaz de } \\
\text { levantarme y comenzar nuevamente. }\end{array}$ & 3 & 1.6 & 3 & 1.6 & 71 & 37.8 & 111 & 59.0 \\
\hline $\begin{array}{l}\text { P11. Puedo imaginar fácilmente muchas } \\
\text { maneras de satisfacer una necesidad. }\end{array}$ & 3 & 1.6 & 10 & 5.3 & 103 & 54.8 & 72 & 38.3 \\
\hline $\begin{array}{l}\text { P12. Soy bastante curioso, por ello } \\
\text { continuamente estoy en búsqueda de } \\
\text { descubrimientos. }\end{array}$ & 1 & 0.5 & 24 & 12.8 & 86 & 45.7 & 77 & 41.0 \\
\hline $\begin{array}{l}\text { P13. Soy capaz de imaginar cómo } \\
\text { pueden hacerse funcionar las cosas. }\end{array}$ & 1 & 0.5 & 18 & 9.6 & 98 & 52.1 & 71 & 37.8 \\
\hline $\begin{array}{l}\text { P14. Donde otros ven problemas, yo } \\
\text { veo oportunidades. }\end{array}$ & 3 & 1.6 & 29 & 15.4 & 110 & 58.5 & 46 & 24.5 \\
\hline $\begin{array}{l}\text { P15. No tengo miedo a emprender } \\
\text { oportunidades }\end{array}$ & 6 & 3.2 & 13 & 6.9 & 74 & 39.4 & 95 & 50.5 \\
\hline
\end{tabular}


cuencias y porcentajes. El presente estudio se apegó a lo dispuesto en el Reglamento de la Ley General de Salud en Materia de Investigación para la Salud ${ }^{(15)}$.

\section{RESULTADOS}

En el estudio participaron 188 estudiantes, de los cuáles $77.1 \%$ fueron mujeres, $50.5 \%$ refirieron tener experiencia laboral. Las edades se encontraron en un rango de 18 a 26 años de edad.

En la tabla 1, se muestran los resultados obtenidos sobre las características emprendedoras, donde se observa que cuatro de cada 10 participantes están de acuerdo en tener confianza de poder alcanzar el éxito en sus proyectos y apuntan a la excelencia en cada cosa que hacen, son curiosos y se encuentra en búsqueda de descubrimientos, les gustan los retos al emprender un nuevo proyecto. Además cinco de cada 10 refieren ver oportunidades donde otros ven problemas, tienen facilidad de imaginar las formas de satisfacer una necesidad y cómo pueden hacer funcionar las cosas. Asimismo, seis de cada 10 intentan aprender lecciones de los errores, dan lo mejor en lo que hace, dicen que todo es posible si se creen en hacerlo, son capaces de levantarse y volver a comenzar después de un error.

\section{DISCUSIÓN}

El presente estudio permitió conocer las características emprendedoras de los estudiantes. Los resultados en cuanto a la confianza de poder alcanzar el éxito en sus proyectos fue diferente a lo reportado en estudios previos ${ }^{(7)}$, quienes encontraron que cinco de cada 10 tienen confianza en el éxito de sus proyectos. Aquellos estudiantes que se consideraron capaces para el emprendimiento son más proactivos y manifiestan sentirse capaces de emprender nuevos proyectos resultado similar a lo enunciado en un estudio relacionado(5), quienes obtuvieron que los estudiantes que experimentan mayor voluntad de emprender fueron quienes consideraban tener los conocimientos, habilidades y experiencia para poner en marcha un nuevo negocio. Los estudiantes consideran que es factible lograr una actividad de emprendimiento y además poseen inclinación a no desaprovechar las oportunidades, resultado similar a lo reportado en investigación previa ${ }^{(11)}$, quienes mencionan que las características emprendedoras promueven una actitud emancipación.

\section{CONCLUSIONES}

Los resultados dan cuenta de las características emprendedoras de los participantes entre las que se destacan tener confianza en sus proyectos, la excelencia en cada cosa que hacen, búsqueda de descubrimientos, el gusto por los retos aprender lecciones de los errores todo es posible si se creen en hacerlo. Por tal motivo, este estudio da indicios del énfasis que debe tener la formación de los profesionales de enfermería orientado al emprendimiento.

Las implicaciones de los resultados obtenidos en el estudio permiten trabajar transversalmente las características de emprendimiento de los estudiantes, dado que evidenciaron la formación de profesionales de enfermería, por lo que aquellos aspectos que favorecen el emprendimiento tales como: motivar a los jóvenes a que se sientan capaces de emprender nuevos proyectos, vencer sus miedos y temores a fracasar, tener una actitud proactiva, contribuye de forma positiva al hecho de que se conviertan en personas emprendedoras.

\section{REFERENCIAS BIBLIOGRÁFICAS}

1. Sánchez-Cañizares SM, Rodríguez-Gutiérrez P. Fomento del emprendimiento universitario mediante la innovación docente en la asignatura Creación de Empresas. International Journal of Educational Research and Innovation (IJERI). 2015;4(1):41-50.

2. Hernández Ruíz KE, González Tamayo LA, Herrera Avilés M. Emprendimiento universitario: generalidades y primeros resultados del taller de sensibilización para profesores sobre espíritu emprendedor [Internet]. Monterry: Instituto Tecnológico y de Estudios Superiores de Monterrey; 2015 [Citado 26 Febrero 2018]. 86 p. Disponible en: https://www. editorialdigitaltec.com/materialadicional/emprendimientouniversitario.pdf

3. Juliá JF. Tribuna de opinión: Empoderamiento y universidad. Una referencia al caso de España y a la UPV. REVESCO: Revista de Estudios Cooperativos. 2014;113(1):7-27.

4. Guerrero M, Urbano D, Ramos AR, Ruiz-Navarro J, Neira I, Fernández-Laviada A. Observatorio de Emprendimiento Universitario en España [Internet]. Madrid: Crue Universidades Españolas-RedEmprendia-CISE; 2016 [Citado 27 Febrero 2018]. 116 p. Disponible en: https://www.crue.org/Documentos\%20compartidos/Publicaciones/Observatorio\%20 de\%20Emprendimiento\%20Universitario/20161201_Observatorio\%20de\%20Emprendimiento\%20Universitario_informe_web.pdf

5. Soria-Barreto K, Zuniga-Jara S, Ruiz-Campo, S. Educación e Intención Emprendedora en Estudiantes Universitarios: Un Caso de Estudio. Formación universitaria. 2016;9(1):25-34.

6. Mussons-Torras M, Tarrats-PonsE. Modelo de credibilidad emprendedora en los estudiantes de enfermería y fisioterapia. Enfermería Global. 2018;49(1):294-308.

7. Durán-Aponte E, Arias-Gómez D. Intención emprendedora en estudiantes universitarios: integración de factores cognitivos y socio-personales. Revista Colombiana de Ciencias Sociales. 2015;6(2):320-340.

8. Ferreira JJ, Raposo ML, Rodrigues RG, Dinis A, Do Paço A. A model of entrepreneurial intention: An application of the psychological and behavioral approaches. Journal of Small Business and Enterprise Development. 2012;19(3):424-40.

9. Valencia Arias JA, Montoya Restrepo I, Montoya Restrepo A. Factores explicativos de las intenciones emprendedoras en estudiantes universitarios. Espacios. 2015;36(5):7.

10. Erdmann AL, Stein Backes D, Alves A, Albino AT, Farias F, Guerini IC, et al. Formación de emprendedores en enfermería: promover capacidades y aptitudes sociopolíticas. Enfer- 
mería Global. 2009;16:1-9.

11. Backes DS, Zamberlan C, Colomé J, Souza MT, Marchiori MT, Erdmann AL, et al. Interatividade sistêmica entre os conceitos interdependentes de cuidado de enfermagem. Aquichan. 2016;16(1):24-31.

12. Novillo Maldonado EF, Sarmiento Chugcho CB, Ollague Valarezco JK, Ramón Ramón DI. Análisis de Actitud Emprendedora en Estudiantes Universitarios: una Perspectiva de Género. INNOVA Research Journal. 2017;2(8):56-63.

13. Arenas Montaño G, Mori FMLV. Una nueva mirada del trabajo profesional de la Licenciada Enfermera. Revista Médica Electrónica Postales Médicos [Internet]. 2015 [Citado 28 Febrero 2018];6(1):1-7. Disponible: https://www.revista-portalesmedicos.com/revista-medica/trabajo-licenciada-enfermera/

14. Roth $E$, Lacoa D. Análisis psicológico del emprendimiento en estudiantes universitarios: medición, relaciones y predicción. Ajayu, 2009;7(1):2-38.

15. Reglamento de la Ley General de Salud en Materia de Investigación para la Salud. Diario Oficial de la Federación, (1984). 